Machtmitteln ausgestattet sind oder - wie der in dieser Hinsicht grundsätzlich gut gerüstete Weltsicherheitsrat - aus Opportunitätsgründen oder wegen einer Veto-Paralyse untätig bleiben.

Summa summarum lehrt die Empirie jedenfalls, dass auch im Feld der internationalen Beziehungen und insbesondere der internationalen (Schieds-)Gerichtsbarkeit bereits heute die unabdingbare Kompetenzausstattung fallweise vorhanden, also Realität ist, und keineswegs pauschal in das Reich der
Utopie verwiesen werden kann. Worauf es also ankommt, ist (eben u.a. auf der Grundlage und mit Hilfe dieses empirischen Vergleichs), die Welt - um ein klassisches "geflügeltes Wort « zu variieren - nicht nur zu erklären, sondern zu verändern, auch die Welt der internationalen (Schieds-)Gerichtsbarkeit. Die Basiskriterien für eine effektive internationale (Schieds-)Gerichtsbarkeit sind jedenfalls benannt.

\title{
Der Wegfall des Zivildienstes muss nicht mehr schrecken
}

\author{
Kommission »Europäische Sicherheit und Zukunft der Bundeswehr«am IFSH
}

In Kürze wird der Bundestag über ein »Zweites Gesetz zur Änderung des Zivildienstgesetzes und anderer Gesetze« beraten. Die Überschrift des Gesetzespakets erstaunt ebenso wie die Zuständigkeit in diesem Verfahren. Erstmalig haben bei substanziellen Änderungen im Wehrrecht nicht das Verteidigungsministerium und der Verteidigungsausschuss des Deutschen Bundestages, sondern das für den Zivildienst zuständige Familienministerium und der Ausschuss für Familie, Senioren, Frauen und Jugend die Federführung. Offensichtlich geht es bei den Regelungen für die Wehrpflicht inzwischen weniger um den Dienst in der Bundeswehr als um den Dienst der Kriegsdienstverweigerer. Die Bundeswehr stellt sich nämlich bereits seit der Anweisung des Verteidigungsministers vom Januar 2004 an seinen Generalinspekteur, die neue Bundeswehrstruktur so auszurichten, dass sie auf Grundwehrdienstleistende nicht länger angewiesen ist, auf den Wegfall der Wehrpflicht ein.

Auch der Wegfall des Zivildienstes - als Folge dieser Entscheidung - hat längst seinen Schrecken verloren. Zwischen Staat und Wohlfahrtsverbänden wird heute praktisch nur noch um die Konditionen des Übergangs gerangelt. Vor allem geht es um die finanzielle Unterstützung der Umstellung, also konkret darum, wie die bisher für den Zivildienst ausgegebenen Haushaltsmittel des Bundes für die sozialen Dienstleistungen erhalten bleiben können, und um den Zeitpunkt. Überraschend haben im Januar einige Wohlfahrtsverbände das Jahr 2008 als Zeitpunkt des Ausstiegs aus Wehrpflicht und Zivildienst genannt. Bis dahin sei eine reibungslose Umstellung möglich. Die Bundesregierung hat diesen Termin nicht bestätigt, aber eben auch nicht dementiert. Als Fixpunkt für Planungen scheint er deshalb sehr nützlich zu sein.

Renate Schmidt ist durch das anstehende Gesetzgebungsverfahren unversehens federführende Ministerin bei der
Novellierung des Wehrpflichtgesetzes geworden. Dabei ist sie eine ausgewiesene Wehrpflichtgegnerin. »Ich halte die Wehrpflicht für überholt, sie ist nicht mehr länger das richtige Modell für Deutschland«, schrieb sie im Juni 2003 in einem Gastkommentar für die Financial Times Deutschland. Als Bundesministerin für Familie, Senioren, Frauen und Jugend ist sie aber für den Zivildienst zuständig und verantwortet weit mehr Einberufungen im Rahmen der Wehrpflicht als der Verteidigungsminister. Während der Verteidigungsminister in diesem Jahr 73.500 Dienstposten mit 83.000 Grundwehrdienstleistenden und freiwillig länger dienenden Wehrdienstleistenden besetzen will, plant die Familienministerin für 93.000 Dienst-. plätze über 120.000 Einberufungen. Dabei hat die jetzige Bundesregierung die "größtmögliche Gerechtigkeit und Gleichbehandlung« von Wehr- und Zivildienstpflichtigen als Maxime im Koalitionsvertrag von 2002 festgeschrieben. Wenn rund 50 Prozent mehr zum Zivildienst als zum Grundwehrdienst einberufen werden, ist die Verletzung der den Kriegsdienstverweigerern zugesagten Gleichbehandlung mehr als offensichtlich.

Während Renate Schmidt Schwierigkeiten hat, mit der schnellen Reduzierung von Dienstposten für Grundwehrdienstleistende Schritt zu halten und sich so die Frage nach der Einberufungsgerechtigkeit stellt, ist die Frage der Wehrgerechtigkeit längst aus dem Ruder gelaufen. Zielvorgabe für die Restwehrpflicht ist die Einberufung von rund 55.000 Wehrpflichtigen pro Jahr. Bei Jahrgängen von durchschnittlich etwa 415.000 Männern wird auf den ersten Blick schon deutlich, dass das Gebot der Gleichbehandlung, unter das das Bundesverfassungsgericht die Durchführung der Wehrpflicht gestellt hat, nicht erfüllt werden kann. So ist heute unbestritten, dass von den tauglich gemusterten und für den Grundwehrdienst verfügbaren Wehrpflichtigen nur noch jeder Zweite einberufen werden kann. Die Wehrverwaltung findet immer neue Ausnahmeregelungen, um den Überschuss an Wehrpflichtigen sozialverträglich verwalten zu können, beispielsweise durch die 
Freistellung Verheirateter oder von Vätern. Mit der beabsichtigten Streichung des Tauglichkeitsgrades 3 werden von jedem Geburtsjahrgang rund 33.000 eigentlich taugliche Wehrpflichtige und noch einmal ebenso viele eigentlich taugliche Zivildienstpflichtige einfach für »dauernd nicht wehrdienstfähig « erklärt mit der Folge, dass sie keinen Dienst mehr leisten müssen. Gerechter wird die Wehrpflicht dadurch allerdings nicht.

Der Zivildienst stellt mit 93.000 Arbeitskräften bei den sozialen Dienstleistern eine scheinbar beachtliche Mitarbeitergruppe, die aber sofort an Gewicht verliert, wenn man weiß, dass allein bei den Wohlfahrtsverbänden 1,2 Millionen Menschen arbeiten. Insgesamt machen Zivildienstleistende rund fünf Prozent der Mitarbeiter in staatlichen und wohlfahrtsverbandlichen sozialen Einrichtungen aus. Vor fünf Jahren (Mitte 1999) waren sogar über 150.000 Zivildienstleistende im Dienst. Der Abbau von knapp 60.000 Zivildienstleistenden war möglich und gelang durch den Einsatz anderer Arbeitskräfte. Öffentlich wurde und wird über diese gelungene Konversion aber nur wenig geredet.

\section{Vorschläge für Ausstiegsszenarien aus dem Zivildienst}

Im Januar 2004 übergab die Kommission »Impulse für die Zivilgesellschaft « ihren Bericht "Perspektiven für Freiwilligendienste und Zivildienst in Deutschland «. Rund 100 Fachleute aus Bundes- und Landesministerien, aus Wohlfahrtverbänden und Freiwilligendiensten hatten ein dreiviertel Jahr lang über die Zeit »nach der Wehrpflicht« beraten und dann Vorschläge unterbreitet, die den Übergang zur wehrpflichtfreien Gesellschaft möglich machen. Einvernehmen bestand und besteht bei allen Fachleuten - auch bei denen der Wohlfahrtsverbände -, dass soziale Dienstleister auf Zivildienstleistende als Hilfskräfte nicht angewiesen sind.

Nach den Empfehlungen der Kommission sollen bis zum Wegfall der Wehrpflicht - und in der Folge: des Zivildienstes - Korrekturen am heutigen Zivildienst vorgenommen werden, um den Übergang zu erleichtern. So wird die Zivildienstdauer an die Wehrdienstdauer angepasst, und der Zivildienst soll zu einem »Lerndienst « weiterentwickelt werden. Dabei sollen die Ausbildungsanteile im Zivildienst dem Umfang der Ausbildungselemente der Freiwilligendienste angeglichen werden.

Von großer Bedeutung ist das öffentliche Eingeständnis, dass Zivildienstleistende nicht »billige Arbeitskräfte« sind, sondern dass ein besetzter Zivildienstplatz pro Jahr rund 15.000 Euro kostet. Davon tragen der Bund gut 8.000 und die Einsatzstellen knapp 7.000 Euro. Seit langem herrscht Einigkeit darüber, dass bei einem Ersatz der Zivildienstleistenden durch reguläre Dauerarbeitskräfte ein Verhältnis von zwei zu drei angenommen werden muss, zwei Dauerarbeitskräfte ersetzen drei Zivildienstleistende. Ein solches Verhältnis ist möglich, weil durch die alle neun Monate wechselnden Zivildienstleistenden erhebliche Arbeitszeit für Einarbeitung, Lehrgänge und fachliche Anleitung verloren geht, die bei Dauerarbeitskräften nur einmalig anfällt. 90.000 Zivildienstleistende könnten also durch 60.000 Dauerarbeitskräfte ersetzt werden. Auf Zivildienstplätzen arbeiten - auch das wird inzwischen sehr realistisch gesehen - Mitarbeiter ohne einschlägige berufliche Vorbildung und in der Regel ohne Arbeitserfahrung. Die Tätigkeiten der Zivildienst-Arbeitsplätze sind so strukturiert, dass sie innerhalb zweier Monate erlernt werden können. Die bei einem Wegfall des Zivildienstes neu zu schaffenden Arbeitsplätze sind also besonders geeignet für Menschen mit geringer oder gar keiner beruflichen Qualifizierung, also für Menschen, für die auf dem heutigen Arbeitsmarkt kaum Arbeitsplätze angeboten werden.

Während für drei Zivildienstleistende 45.000 Euro im Jahr ausgegeben werden, belaufen sich die Arbeitgeberkosten für eine junge Hilfskraft auf deutlich unter 25.000 Euro pro Jahr. Zivildienstleistende können also durch reguläre Arbeitskräfte ersetzt werden, ohne dass Mehrkosten entstehen - im Gegenteil. Volkswirtschaftlich werden Arbeitslosengeld und Sozialleistungen für 60.000 dann nicht mehr arbeitslose Menschen eingespart.

In der Fachdiskussion wird aber nicht davon ausgegangen, dass alle Zivildienstplätze durch reguläre Dauerarbeitskräfte ersetzt werden. Angenommen wird, dass sich in der Praxis ein »Mix« ergibt aus Vollzeitarbeitsplätzen, Teilzeitarbeitsplätzen und Mini-Jobs, Freiwilligendiensten und ehrenamtlichen Tätigkeiten.

Vollzeitarbeitsplätze werden überall dort entstehen, wo Zivildienstleistende in der Basisversorgung eingesetzt werden, zum Beispiel im Hol- und Bringedienst eines Krankenhauses, in der Hausmeisterei, Küche oder Wäscherei von Altenheimen, in Notrufzentralen usw.

Mini-Jobs und Teilzeitarbeitsplätze dürften eingerichtet werden in Arbeitsbereichen, in denen es zeitliche Arbeitsschwerpunkte gibt, so zum Beispiel bei »Essen auf Rädern « oder im Behindertenfahrdienst usw.

Mitarbeiter und Mitarbeiterinnen aus Freiwilligendiensten werden vermutlich dort tätig werden, wo auch Zivildienstleistende bisher interessante Lernfelder vorfanden, zum Beispiel in der so genannten ISB-K, der individuellen Schwerstbehindertenbetreuung bei Kindern (Begleitung von behinderten Kindern in Regelkindergärten und Regelschulen), in Umwelteinrichtungen oder für Tätigkeiten, »die ein bisschen Farbe in das Grau des Heim- oder Krankenhauslebens bringen « wie Besuchs-, Vorleseund Veranstaltungsbegleitdienste usw.

Ehrenamtliche werden an den Stellen Zivildienstleistende ersetzen können, an denen echte Zusatztätigkeiten übernommen wurden, so zum Beispiel der Baumschnittkurs eines Umweltverbandes, die Theaterbegleitung in einer Alteneinrichtung usw.

Es ist zu erwarten, dass die Umstellung auf diesen Mix an »Ersatzkräften« wie schon die bisherige Reduzierung des Zivildienstes fast geräuschlos über die Bühne gehen wird. Und in Wirklichkeit handelt es sich um die Rückkehr zur Normalität einer freiheitlichen Gesellschaft, die auf freiwillige Mitarbeit setzt. 


\section{Klares Nein zur allgemeinen Dienstpflicht}

Im Zusammenhang mit dem möglichen Wegfall des Zivildienstes haben die Ministerpräsidenten von Nordrhein-Westfalen und Sachsen-Anhalt sich in der BildZeitung für die Einführung einer allgemeinen Dienstpflicht ausgesprochen. Ob die Ministerpräsidenten nun der Beobachtung durch den Verfassungsschutz zu unterstellen wären, sei dahingestellt. Zumindest sind ihre Stellungnahmen eindeutig nicht durch die Verfassung der Bundesrepublik Deutschland gedeckt.

Artikel 12 Grundgesetz verbietet die Einführung eines sozialen Pflichtjahres: »Niemand darf zu einer bestimmten Arbeit gezwungen werden.« Aber auch eine Änderung des Grundgesetzes - möglich nur mit einer ZweidrittelMehrheit - würde die Einführung eines sozialen Pflichtjahres in Deutschland nicht ermöglichen. Internationale Menschenrechtskonventionen gelten in Deutschland wie nationales Recht. Die Bundesrepublik Deutschland müsste vor der Einführung eines »sozialen Pflichtjahres« die Geltung der Menschenrechtserklärung der Vereinten Nationen, des internationalen Paktes über bürgerliche und politische Rechte von 1966, des Übereinkommens über Zwangs- und Pflichtarbeit vom 26.6.1961, des Übereinkommens über die Abschaffung der Zwangsarbeit vom 25.6.1957, die Konvention zum Schutz der Menschenrechte und Grundfreiheiten des Europarates vom 4.11. 1950 aufheben. Als Ausnahmen sind national wie international nur der Zwang zum Wehrdienst sowie in Strafverfahren festgelegte Arbeitsauflagen zugelassen. Der Vorschlag, dass ausgerechnet unser Land mit seiner historischen Belastung sich über das Verbot von Zwangs- und Pflichtdiensten hinwegsetzt, ist geschichtsvergessen und eine Absage an die zivilisierte Welt. Naturalleistungen zwangsweise einzufordern ist ein Rückfall in Zeiten der mittelalterlichen Frondienste. Derzeit gibt es ein solches Pflichtjahr weltweit nur im diktatorischen Burma.

Aber auch ganz praktische Überlegungen zeigen schnell, dass die Vorschläge der Ministerpräsidenten völlig ungeeignet sind, den Zivildienst zu ersetzen: Völlig aus dem Blick gerät üblicherweise die Größenordnung eines »sozialen Pflichtjahres«. Rund 800.000 Männer und Frauen umfasst ein durchschnittlicher Geburtsjahrgang. Rund 100.000 dürften - aus welchen Gründen auch immer für eine Dienstpflicht nicht in Frage kommen. Rund 100.000 könnte die Bundeswehr und rund 100.000 der bisherige Zivildienst aufnehmen, da es im Falle der Einführung einer allgemeinen Dienstpflicht wohl bei der faktischen Beibehaltung der Wehr- und Zivildienstpflicht bleiben würde. Wer ein »Soziales Pflichtjahr« fordert, muss also ein Organisationsmodell entwickeln, das pro Jahr 500.000 junge Erwachsene zusätzlich zum bestehenden Wehr- und Zivildienst für einen Zeitraum von rund einem Jahr unterbringt.

Wenn etwas »verpflichtend « ist, muss gleichzeitig kontrolliert werden, dass alle das »soziale Pflichtjahr « auch wirklich machen. Staatliche Pflichtdienste bedeuten zum einen im Umkehrschluss auch staatliche Fürsorge (Kran- kenschutz, Haftpflicht etc.) mit den entsprechenden Verwaltungen. Zum anderen müssen Sanktionen für diejenigen vorgesehen werden, die der Pflicht nicht nachkommen. Geldstrafen kommen dabei nicht in Frage, weil sie für Reiche ein »Freikaufen « ermöglichen würden. Es bleibt wie beim Wehrdienst nur die angedrohte Freiheitsstrafe als adäquates Sanktionsmittel.

Junge Erwachsene dürften im »Sozialen Pflichtjahr« etwa so viel kosten wie die heutigen Grundwehr- und Zivildienstleistenden oder wie die MitarbeiterInnen im Freiwilligen Sozialen Jahr. Rund 15.000 Euro werden für diese Pflicht- und Freiwilligendienste pro Person und Jahr veranschlagt. Getragen wird dieser Betrag bisher je nach Dienstart zu unterschiedlichen Anteilen von den Einrichtungen und über die öffentliche Hand. Ein »soziales Pflichtjahr für alle« würde 7,5 Milliarden Euro (500.000 Dienstpflichtige mal 15.000 Euro und Jahr) kosten, zusätzlich zu dem Geld, was heute schon für Grundwehrdienst und Zivildienst ausgegeben wird.

Mit den 7,5 Milliarden Euro, die ein Pflichtjahr kosten würde, ließen sich rund 300.000 neue tariflich bezahlte Arbeitsplätze auf einem dem Zivildienst vergleichbaren Niveau (Hilfs- und Zuarbeiten, rund 25.000 Euro Arbeitgeberbrutto) schaffen. Eine Reduzierung der Arbeitslosenzahlen um 300.000 hätte darüber hinaus eine erhebliche Entlastungswirkung für die Sozialkassen. Oder es könnten auch 150.000 FacharbeiterInnen mit 50.000 Euro Arbeitgeberbrutto neu eingestellt werden.

Ein »soziales Pflichtjahr für alle« verzögert den Berufseinstieg um ein Jahr und verkürzt die erreichbare Lebensarbeitszeit entsprechend. Rentenberechnungsmodelle müssten das entsprechend berücksichtigen. Volkswirtschaftliche Studien gehen davon aus, dass ein um ein Jahr späterer Berufseinstieg einen Einkommensnachteil von gut 90.000 Euro - bezogen auf die Lebensarbeitszeit - ausmacht. Entsprechend geringer sind dann auch die Leistungen in die Sozialversicherungskassen.

Die Fachdiskussion ist sich in der Frage der Bewertung von Pflichtdiensten weitgehend einig. Sie werden als völlig ungeeignet abgelehnt. »Die Einführung einer allgemeinen Dienstpflicht anstelle der Wehrpflicht durch Verfassungsänderung bzw. die Einbeziehung junger Frauen in die allgemeine Wehrpflicht hält die Kommission nicht nur für völkerrechtswidrig, sondern für einen grundsätzlich falschen Weg, Eigeninitiative, Mitgestaltung und Beteiligung aller Altersgruppen in der Zivilgesellschaft zu fördern «, schreibt die Kommission »Impulse für die Zivilgesellschaft« quasi als Präambel zu ihrem Bericht.

\section{Blick zu den europäischen Nachbarn}

In verschiedenen Nachbarländern Deutschlands wurde in den letzten Jahren die Wehrpflicht und damit auch der Zivildienst - im Umfang dort aber jeweils deutlich kleiner als in Deutschland - abgeschafft. Die Diskussion um den Umbau sozialer Dienstleistungen und deren Finanzierung fand und findet in diesen Ländern ebenso wie in Deutschland statt. Dabei spielte der wegfallende Zivildienst aber nur eine völlig untergeordnete Rolle. Bedeutsamer war die Diskussion, welche Angebote Jugendlichen gemacht werden können, um ihre Bereitschaft zu freiwilligem Engagement aufzugreifen. Dass diese Diskussion aber weitgehend unabhängig von der Diskussion um die 
Wehrpflicht stattfindet, zeigt das Beispiel Englands, das seit 1957 keine Wehrpflicht mehr hat. Frankreich, Belgien, die Niederlande und England sind zu unterschiedlichen Lösungen gekommen, meistens einem »Mix« aus Maßnahmen zur Bekämpfung von Jugendarbeitslosigkeit und gezielten Angeboten an Freiwilligendiensten. Letztlich spielte der Wegfall des Zivildienstes für soziale Dienstleister und Gesellschaft keine große Rolle, weil Problembereiche wie Jugendarbeitslosigkeit und Lösungen zur Verbesserung freiwilligen sozialen Engagements die weit wichtigeren gesellschaftspolitischen Fragestellungen sind.

\section{Fazit}

Die Kommission »Europäische Sicherheit und Zukunft der Bundeswehr« begrüßt die Weichenstellung für den Wegfall des Zivildienstes. Weder gibt es heute noch eine tragfähige sicherheitspolitische Begründung für die Beibehaltung der Wehrpflicht, noch erweist es sich als nötig, an der Verpflichtung junger Männer zu Ersatzdiensten weiter festzuhalten. In einem europäischen Sicherheitssystem sind Zwangsdienste entbehrlich. Die Mehrheit der europäischen Staaten hat diese Erkenntnis bereits umgesetzt. Nun ist Deutschland am Zug.

\section{Jochen Rasch}

\section{Völkerrecht/Vereinte Nationen}

Ambos, Kai/Arnold, Jörg (Hrsg.): Der Irak-Krieg und das Völkerrecht. Berlin (Berliner Wissenschafts-Verlag) 2004.

Fenton, Neil: Understanding the UN Security Council. Coercion or Consent? Aldershot (Ashgate) 2004.

Kirton, John J./Stefanova, Radoslava N. (Hrsg.): The G8, the United Nations, and the Conflict Prevention. Aldershot (Ashgate) 2004.

Schweitzer, Michael/Weber, Albrecht: Handbuch der Völkerrechtspraxis der Bundesrepublik Deutschland. BadenBaden (Nomos) 2004.

Malone, David M. (Hrsg.): The UN Security Council From the Cold War to the 21st century. Boulder/Colo. (Rienner) 2004 .

Schaller, Christian: Das Friedenssicherungsrecht im Kampf gegen den Terrorismus. Gewaltverbot, Kollektive Sicherheit, Selbstverteidigung und Präemption. Berlin (SWP), 2004.

\section{Abrüstung/ Rüstungskontrolle/ Militär/Verteidigung}

Howard, Roge: Iran in Crisis?: Nuclear Ambitions and the American Response. London (Zed Books) 2004.

Kutz, Martin: Innere Führung in der Bundeswehr: Auf andere Streitkräfte übertragbar?. Hamburg (Führungsakademie der Bundeswehr, Fachbereich Sozialwissenschaften) 2004.

Schmidt, Hans-Joachim: Der Wandel der konventionellen Rüstungskontrolle 1989-1996. Frankfurt am Main (Campus) 2004.

Squassoni, Sharon A.: Weapons of mass destruction: trade between North Korea and Pakistan. Washington (LoC) 2004.

Thraenert, Oliver: Verliert die Verbreitung von Kernwaffen ihren Schrecken?: die neuesten Entwicklungen in Iran, Libyen, Nordkorea und Pakistan. Berlin (SWP) 2004.

Brahimi, Lakhdar: The political transition in Iraq. Report of the fact-finding mission. New York (N.Y.) 2004.

\section{Nationalismus/ethnische Konflikte}

Akçam, Taner: From Empire to Republic: Turkish Nationalism and the Armenian Genocide. London (Zed Books) 2004.

Bertrand, Jacques: Nationalism and Ethnic Conflict in Indonesia. Cambridge (Cambridge UP) 2004.

Brown, Nathan J.: Palestinian Politics after the Oslo Accords: Resuming Arab Palestine. Berkeley (Univ. of California Pr.) 2003.

Hays Gries, Peter: Chinas New Nationalism: Pride, Politics, and Diplomacy. Berkeley (Univ. of California Pr.) 2004.

Jones, Adam (Hrsg.): Genocide, War Crimes, and the West: History and Complicity. London (Zed Books) 2004.

Kaußen, Stephan: Von der Apartheid zur Demokratie: Die politische Transformation Südafrikas. Wiesbaden (Westdeutscher Verlag), 2003.

Michel Wieviorka: Kulturelle Differenzen und kollektive
Identitäten. Hamburg (Hamburger Edition) 2003.

Naimark, Norman M.: Flammender Hass: Ethnische Säuberungen im 20. Jahrhundert. München (C.H. Beck) 2004.

Roberg, Robert I.: When States Fail: Causes and Consequences. Princeton (Princeton UP) 2004.

Schetter, Conrad: Ethnizität und ethnische Konflikte in Afghanistan: rationale und irrationale Aspekte in nationalen Optionen. Berlin (Reimer) 2003.

Verbeek, Bernhard: Die Wurzeln der Kriege: Zur Evolution ethnischer und religiöser Konflikte. Stuttgart (S. Hirzel) 2003.

Zagorin, Perez: How the Idea of Religious Toleration Came to the West. Princeton (Princeton UP) 2003.

\section{Europa/EU/Ost- erweiterung}

Biskupski, M. B. B. (Hrsg.): Ideology, Politics and Diplomacy in East Central Europe. 